\title{
Sudden Sensorioneural Hearing Loss and Autoimmune Systemic Diseases
}

\author{
Bruno Almeida Antunes Rossini ${ }^{1}$ Norma de Oliveira Penido ${ }^{1} \quad$ Mario Sergio Lei Munhoz ${ }^{1}$ \\ Eduardo Amaro Bogaz ${ }^{1}$ Renata Souza Curi ${ }^{1}$ \\ 1 Department of ENT, Universidade Federal de São Paulo, \\ São Paulo, Brazil \\ Address for correspondence Bruno Almeida Antunes Rossini, MD, \\ Clinica Otovita de Otorrinolaringologia, Rua Cubatão, 86-1407, Sao \\ Paulo 04013000, Brazil (e-mail: baarossini@gmail.com).
}

Int Arch Otorhinolaryngol 2017;21:213-223.

\begin{abstract}
Introduction Several authors have demonstrated the relationship between sudden sensorineural hearing loss (SNHL) and systemic autoimmune diseases (SAD). Immunemediated SNHL can rarely present as unilateral sudden SNHL and manifests itself in the contralateral ear only after years. It presents clinical relevance for being one of the few SNHL that may be reversible given that early and appropriate treatment is applied.

Objective The objective of this study is to describe the clinical presentations and audiological findings from patients with idiopathic sudden SNHL and SAD associated with a probable diagnosis of immune-mediated SNHL. Furthermore, we strive to estimate the prevalence of SAD in patients with sudden SNHL.

Methods This is an observational retrospective cohort. We have selected and studied patients with SAD. Revision of available literature on scientific repositories.

Results We evaluated 339 patients with sudden SNHL. Among them, 13 (3.83\%) patients suffered from SAD. Three patients had bilateral involvement, a total of 16 ears. We evaluate and describe various clinical, epidemiological, and audiological aspects of this sample.

Keywords

- hearing loss

- sensorineural

- hearing loss

- sudden

- autoimmune diseases

- humans

Conclusion In our sample of patients with sudden SNHL, the prevalence of SAD was found relevant. The majority had tinnitus and dizziness concomitant hearing loss, unilateral involvement and had experienced profound hearing loss at the time of the installation. In spite of instituted treatment, most cases showed no improvement in audiometric thresholds. Apparently, patients with sudden SNHL and SAD have a more severe initial impairment, higher percentage of bilateral, lower response to treatment, and worse prognosis than patients with sudden SNHL of unknown etiology.
\end{abstract}

\section{Introduction}

In recent years, several authors have tried to demonstrate the relationship between sudden sensorineural hearing loss (SNHL) and systemic autoimmune diseases (SAD), seeking to elucidate a possible immune-mediated etiology involved in cases of sudden hearing loss. ${ }^{1-5}$

Sudden SNHL was first described by De Kleyn et al in 1944, and the most widely used definition is based on audiological

received

June 15, 2015

accepted

June 3, 2016

published online

July 26, 2016 and temporal parameters, which predict fall of hearing threshold by bone conduction at least $30 \mathrm{~dB}$ in three contiguous frequencies in a period ranging from a few minutes to 72 hours. Its incidence ranges from 5 to 20 individuals per 100,000 people per annum. ${ }^{6,7}$

In $70 \%$ to $90 \%$ of cases of sudden SNHL, the etiology is not identified. Thus, this percentage of cases is classified as idiopathic sudden SNHL. Theories as to the cause of injury
Copyright $\odot 2017$ by Thieme Revinter

Publicações Ltda, Rio de Janeiro, Brazil
License terms

()(1) $\Theta \circledast$ 10.1055/s-0036-1586162. ISSN $1809-9777$. 
in such situations point to vascular injury, rupture of membranes, viral or bacterial infection, and immune mediated injury. $^{8-12}$

Immune-mediated SNHL usually manifests as a rapidly progressive sensorineural loss, bilateral and asymmetrical. Even today, it remains as a challenging disease. ${ }^{13}$

In 1958 , Lehnhardt ${ }^{14}$ was the first to suggest the possibility of sudden or rapidly progressive hearing loss to be the result of an autoimmune process against the inner ear. Schiff and Brown ${ }^{15}$ in 1974 speculated that, due to the improvement of patients with SSNHL following the use of adrenocorticotropic hormone, its etiology should be an autoimmune vasculitis. In 1979, McCabe ${ }^{16}$ reported cases of sudden SNHL loss successfully treated by immune suppressive therapy and introduced the clinical entity: autoimmune hearing loss.

Although the pathophysiology of immune-mediated SNHL remains unknown, the positive response to immunosuppressive therapy and corticosteroid reinforces the existence of immune-mediated mechanisms. Given that the damage to the inner ear can occur not only by reaction antigen autoantibody, but also by various other mechanisms such as activation of the complement system, direct action of cytotoxic $\mathrm{T}$ cells, among others, experts believe that the term immune-mediated PANS is more appropriate than autoimmune hearing loss. ${ }^{17,18}$

In $30 \%$ of cases, immune-mediated SNHL may be associated with the SAD. This association is prominent in the following diseases: systemic lupus erythematosus (SLE), antiphospholipid syndrome, Susac syndrome, polyarteritis nodosa, rheumatoid arthritis (RA), Wegener's granulomatosis, Sjögren's syndrome, Behcet's syndrome, sarcoidosis, Cogan syndrome, among others. However, the incidence of involvement of the inner ear in the SAD varies greatly. ${ }^{19,20}$

Immune-mediated SNHL most often affects female patients between their thirties and fifties. It is estimated to account for less than $1 \%$ of hearing loss - a small percentage, but it is difficult to assess its impact due to the lack of tests that define this etiology. Cases of sudden SNHL with immune-mediated etiology are even rarer and difficult to diagnose. ${ }^{17,18}$

In most cases, the clinical picture of immune-mediated SNHL is characterized by sensorineural hearing loss, bilateral, asymmetric and rapidly progressive, often associated with tinnitus and vestibular symptoms. An important feature is its fast progression: it can lead to severe bilateral SNHL in a few days or weeks. In rare cases, it presents as unilateral sudden SNHL and manifests only in the contralateral ear after a variable number of days up to years. ${ }^{13,17}$ The diagnosis is eminently based on the clinical picture.

Anamnesis is essential to provide data regarding the existence of associated systemic diseases and the ENT exam commonly shows no alterations. ${ }^{18}$

Pure tone audiometry does not reveal typical curve pattern and speech discrimination cannot present proportional to pure tone thresholds. ${ }^{17}$ Imaging tests such as magnetic resonance (MR) imaging and positron emission tomography can demonstrate inflammatory activity in the inner ear, and thus contribute to the diagnosis. ${ }^{21,22}$ There are no appropriate laboratory tests for the diagnosis of certainty yet. ${ }^{18,19,23}$
The positive response to the therapeutic treatment with corticosteroids and audiogram worsening in medication dose reduction attempts to reinforce the suspicion that etiology. ${ }^{19}$ The suspicion of the disease is fundamental for the diagnosis of immune-mediated SNHL, especially in cases of atypical and/or evolution with systemic comorbidities. Early treatment is crucial for a better prognosis. ${ }^{17}$

The clinical importance of immune-mediated SNHL largely lies in the fact that it is one of the few sensorineural hearing losses that may be reversible with early and adequate treatment. $^{13,17}$

The objective of this study is to describe the different audiological and epidemiological clinical presentations of patients with idiopathic sudden SNHL and associated systemic autoimmune disease, who were diagnosed with probable immune-mediated SNHL. Another aim was to estimate the prevalence of systemic autoimmune diseases in patients with sudden SNHL.

\section{Methods}

The project was an observational retrospective cohort study. Our sample consisted of patients experiencing sudden SNHL, from the SSNHL clinic of a tertiary university hospital. These patients' medical records were followed from 2000 up to 2012. This study received approval from the Ethics Committee of the institution, under the registration number 0632/11.

\section{Inclusion and Exclusion Criteria}

The study included all patients with idiopathic sudden SNHL of at least $30 \mathrm{~dB}$ in at least three consecutive frequencies, over a period of 72 hours.

Then, we selected and separately analyzed patients with SAD, which we diagnosed by clinical criteria and specific rheumatologic laboratory tests for different diseases.

The study excluded patients with sudden SNHL with defined etiologies, such as tumors, trauma, multiple sclerosis, and otitis.

\section{Medical Records Review}

We analyzed the medical records of patients who met the inclusion criteria for clinical data on: age and date of birth; gender; start date of hearing loss; concomitant symptoms such as tinnitus, vertigo, ear pain, and ear fullness; presence of comorbidities, such as hypertension, diabetes, thyroid changes and habits; use of medications; family history; audiometric parameters; and imaging tests.

The patients' hearing tests were performed with a standard audiometer (MA-41, MAICO, Eden Prairie, U.S.A.). This included tonal and vocal audiometry, with a threshold search and speech recognition index. We evaluated the initial and final audiometric parameters, the latter being obtained at least two months after the initial audiometry, or earlier in case of complete recovery. Patients also underwent blood testing that included complete blood count, serum fasting glucose, lipid dosage, renal function and thyroid function, erythrocyte sedimentation rate and serology for syphilis, AIDS, borreliosis when deemed necessary. The majority of 
patients also underwent imaging (MR brain). Patients suspected of SAD underwent specific serological tests determined by the rheumatology team, which could specify the suspected immune-mediated disease.

\section{Audiometric Curves' Classification}

For the classification of audiometric curves, we considered the following criteria: the bass frequencies corresponding to 250 and $500 \mathrm{~Hz}$, the average comprising 1 and $2 \mathrm{kHz}$, and acute, 3 to $8 \mathrm{kHz}$. Upward curves were those with a decrease greater than $15 \mathrm{~dB}$ at worst severe frequency relative to other frequencies; curve " $U$ " with those reductions greater than $15 \mathrm{~dB}$ in the worst average frequency, compared with the worst low and high frequencies; curve "inverted U" when there is a decrease greater than $15 \mathrm{~dB}$ in the worst low and high frequencies compared with the average; downslope when there is a reduction of $15 \mathrm{~dB}$ in the arithmetic average of 4 to $8 \mathrm{kHz}$ with respect to the arithmetic average of the frequencies 250 and $500 \mathrm{~Hz}$; and finally, flat, when there is less than $15 \mathrm{~dB}$ difference between the averages of 250 and $500 \mathrm{~Hz}, 1$ and $2 \mathrm{kHz}$ and 4 to $8 \mathrm{kHz}$.

\section{Intensity Degree Classification of Hearing Loss}

We categorized the intensity of hearing loss into four degrees.

When the arithmetic means of pure tones (PTA) was below $25 \mathrm{~dB}$, we did not consider it a hearing loss; between 26 and $40 \mathrm{~dB}$ we considered it mild. Moderate hearing loss was defined between 41 and $70 \mathrm{~dB}$, severe hearing loss between 71 and $90 \mathrm{~dB}$, and profound above $90 \mathrm{~dB}$.

\section{Arithmetic Means of Pure Tones Obtained}

We obtained arithmetic means from initial and final pure tone in all patients using the following methodology: arithmetic average of pure tone for each patient in accordance with the group of frequencies affected. When low and middle frequencies were hit, we obtained the arithmetic mean of the frequencies of $0.25,0.5,1$ and $2 \mathrm{kHz}$; medium and high when the average of the frequencies of 1, 2, 3, 4, 6 and $8 \mathrm{kHz}$; when only acute, average 3, 4, 6 and $8 \mathrm{kHz}$; when severe, acute medium and the average of all eight frequencies. When the hearing thresholds of deep losses were not detected, was considered as the maximum response audiometer, in this case, $120 \mathrm{~dB}$.

\section{Hearing Recovery Criteria by Pure Tone Audiometry and Vocal}

- Improvement: change for better on classification of the degree of intensity of hearing loss or improved more than $12 \%$ of the IRF.

- Worsening: change for worse on classification of the degree of intensity of hearing loss worsens or greater than $12 \%$ of the IRF.

- Stable: Maintenance intensity degree classification of the hearing loss and change the IRF less than or equal to $12 \%$.

\section{Results}

We evaluated 339 records of all the patients who were diagnosed with sudden SNHL from the SNHL clinic of our institution, from 2000 up to 2012 . We analyzed the results by separating the sample with only SSNHL without SAD, and SAD associated patients.

Thirteen patients (3.83\%), besides the sudden SNHL also had a previous diagnosis confirmed by serological and rheumatologic criteria specific SAD exam. Nine (69.23\%) were women and four (30.77\%) men, eight (61.54\%) were Caucasians, and five (38.46\%) afro-descendants. The average age of patients in this sample at the time of sudden SNHL installation was 44 years of age, with a minimum age of 21 and maximum 68. Regarding SAD, four (30.77\%) patients of SLE; four (30.77\%), AR; two (15.38\%), scleroderma; one (7.69\%), Sjogren's syndrome; one (7.69\%), Cogan's syndrome; one (7.69\%), Susac syndrome; one (7.69\%), psoriasis; one (7.69\%), vitiligo and one (7.69\%), ankylosing spondylitis. Three (23.07\%) patients showed more than one concurrent SAD, that is, one had AR and scleroderma; another had Sjogren's syndrome and scleroderma and a third, AR and vitiligo (-Table 1).

With regard to comorbidities at the beginning of followup, six (46.15\%) patients had systemic hypertension (SH); two (15.38\%), smoking; one (7.69\%), hypothyroidism; one (7.69\%), coronary artery disease (CA); one (7.69\%), chronic renal failure (CRF) and one (7.69\%), diabetes mellitus (DM). Three (23.08\%) of these manifested concomitant comorbidities, which means, one was hypertension $\mathrm{SH}$, diabetes mellitus $\mathrm{DM}$ and $\mathrm{AI}$; another one, hypertension $\mathrm{SH}$ and $\mathrm{CRF}$ and the third one, hypertension SH and hypothyroidism (- Table 1).

Besides the sudden SNHL, other audiological symptoms reported by patients were buzzing in thirteen patients (100\%), dizziness in seven patients (53.8\%), and ear fullness in five patients (38.4\%).

Otoscopy test of the thirteen patients was normal. Out of the thirteen patients, ten $(76.92 \%)$ underwent magnetic resonance imaging. None of them showed cochlear enhancement on T1-weighted after contrast injection.

With regard to treatment, we administered a full dose $(1 \mathrm{mg} / \mathrm{kg})$ of systemic corticosteroids prednisone in all 13 (100\%) patients. In addition to the steroids, we also used immunosuppressants as shown in - Table 2.

The time between the sudden SNHL and the start of treatment averaged 21 days, with a minimum of 6 and maximum of 60 days. The average of the follow-up of these patients was 27.6 months, with a minimum of two months and a maximum of 66 months.

In nine (69.23\%) patients, the side initially affected was on the right hand side and in three (23.08\%) was on the left. One patient (7.69\%), whose underlying disease was SLE, expressed simultaneous bilateral involvement. In other two (15.38\%) patients, there was contralateral posterior involvement, approximately 15 days after the first event. One of these patients had underlying disease as Susac syndrome and the other one, Cogan's syndrome.

We observed bilateral cases in three $(23.08 \%)$ out of 13 patients, which means that 16 ears showed symptoms of sudden SNHL in patients with SAD. We analyze below these 16 ears.

The initial graduation of hearing loss (found upon admission to our service) presented as follows: seven (43.75\%) ears 
Table 1 Demographic data and underlying diseases

\begin{tabular}{|l|l|l|l|l|l|}
\hline Case & Age in CN & Gender & Ethnicity & Systemic immune-mediated disease & Comorbidities in the new case \\
\hline 1 & 21 & M & CN & EA & \\
\hline 2 & 68 & F & AD & SLE & SH \\
\hline 3 & 37 & M & CN & SLE & SH/IRC \\
\hline 4 & 47 & F & AD & RA & SH \\
\hline 5 & 26 & M & AD & SD Cogan & \\
\hline 6 & 48 & F & CN & Psoriasis & SH/TH \\
\hline 7 & 25 & M & CN & SLE & \\
\hline 8 & 53 & F & CN & RA + scleroderma & SH/DM/CP \\
\hline 9 & 56 & F & CN & RA & smoking habit \\
\hline 10 & 45 & F & AD & SLE & - \\
\hline 11 & 42 & F & CN & SD Susac & SH \\
\hline 12 & 52 & F & AD & Sd Sjogren + Scleroderma & smoking habit \\
\hline 13 & 54 & F & CN & RA + vitiligo & . \\
\hline
\end{tabular}

Abbreviations: AD, Afro-descendant; AS, Ankylosing Spondylitis; CN, Caucasian; CRF, Chronic Renal Failure; DM, diabetes mellitus; F, Female; HT, Hypothyroidism; M, Male; RA, Rheumatoid Arthritis; Sd, Syndrome; SH, Systemic Hypertension (high blood pressure); SLE, Systemic Lupus Erythematosus; UA, Unstable Angina.

had profound hearing loss; four (25\%), severe hearing loss; three (18.75\%) had moderate hearing loss; and two (12.50\%), mild hearing loss (-Table 3 ). The affected RTS 16 ears performed as follows: ten (62.50\%) with RTS greater than $90 \mathrm{~dB}$; one (6.25\%) with RTS between 71-90 dB; two (12.50\%) with RTS between $26-40 \mathrm{~dB}$; one (6.25\%) with RTS between 41-70 dB; and two (12.50\%), up to $25 \mathrm{~dB}$ (-Table 3). The SRI for monosyllabic words of affected ears presented the following distribution: SRI zero in ten (62.50\%); SRI equal to $45 \%$ in one (6.25\%); SRI equal to $75 \%$ in one (6.25\%); SRI equal to $92 \%$ in 3 (18.75\%); and SRI equal to $96 \%$ in one (6.25\%) (- Table 3 ).

Regarding the initial type of audiometric curve of the 16 affected ears, five (31.25\%) had a flat curve; four (25.00\%), downslope; three (18.75\%), U-type curve, and in four (25.00\%)

Table 2 Medications taken on the treatment

\begin{tabular}{|l|l|l|l|l|l|l|l|l|}
\hline Case & $\begin{array}{l}\text { S. } \\
\text { steroids }\end{array}$ & $\begin{array}{l}\text { I. } \\
\text { steroids }\end{array}$ & Azathioprine & Methotrexate & Cyclophosph. & Chloroquine & Infliximab & $\begin{array}{l}\text { Sufasa } \\
\text { Lazine }\end{array}$ \\
\hline 1 & $\mathrm{Y}$ & $\mathrm{Y}$ & $\mathrm{N}$ & $\mathrm{Y}$ & $\mathrm{N}$ & $\mathrm{N}$ & $\mathrm{Y}$ \\
\hline 2 & $\mathrm{Y}$ & $\mathrm{N}$ & $\mathrm{Y}$ & $\mathrm{N}$ & $\mathrm{N}$ & $\mathrm{N}$ & $\mathrm{N}$ \\
\hline 3 & $\mathrm{Y}$ & $\mathrm{N}$ & $\mathrm{N}$ & $\mathrm{N}$ & $\mathrm{N}$ & $\mathrm{N}$ \\
\hline 4 & $\mathrm{Y}$ & $\mathrm{N}$ & $\mathrm{N}$ & $\mathrm{Y}$ & $\mathrm{N}$ & $\mathrm{N}$ & $\mathrm{N}$ & $\mathrm{N}$ \\
\hline 5 & $\mathrm{Y}$ & $\mathrm{N}$ & $\mathrm{N}$ & $\mathrm{N}$ & $\mathrm{Y}$ & $\mathrm{N}$ & $\mathrm{N}$ & $\mathrm{N}$ \\
\hline 6 & $\mathrm{Y}$ & $\mathrm{N}$ & $\mathrm{N}$ & $\mathrm{N}$ & $\mathrm{N}$ & $\mathrm{N}$ & $\mathrm{N}$ \\
\hline 7 & $\mathrm{Y}$ & $\mathrm{N}$ & $\mathrm{N}$ & $\mathrm{N}$ & $\mathrm{N}$ & $\mathrm{N}$ \\
\hline 8 & $\mathrm{Y}$ & $\mathrm{N}$ & $\mathrm{Y}$ & $\mathrm{Y}$ & $\mathrm{N}$ & $\mathrm{N}$ & $\mathrm{N}$ & $\mathrm{N}$ \\
\hline 9 & $\mathrm{Y}$ & $\mathrm{N}$ & $\mathrm{N}$ & $\mathrm{N}$ & $\mathrm{N}$ & $\mathrm{N}$ \\
\hline 10 & $\mathrm{Y}$ & $\mathrm{N}$ & $\mathrm{N}$ & $\mathrm{Y}$ & $\mathrm{N}$ & $\mathrm{N}$ & $\mathrm{N}$ \\
\hline 11 & $\mathrm{Y}$ & $\mathrm{N}$ & $\mathrm{N}$ & $\mathrm{N}$ & $\mathrm{Y}$ & $\mathrm{N}$ \\
\hline 12 & $\mathrm{Y}$ & $\mathrm{N}$ & $\mathrm{N}$ & $\mathrm{N}$ & $\mathrm{N}$ & $\mathrm{N}$ \\
\hline 13 & $\mathrm{Y}$ & $\mathrm{N}$ & $\mathrm{N}$ & $\mathrm{Y}$ & $\mathrm{N}$ & $\mathrm{N}$ & $\mathrm{N}$ \\
\hline Total & 13 & 1 & 2 & 15.38 & 38.46 & 15.38 & $\mathrm{~N}$ & $\mathrm{~N}$ \\
\hline Percentage $(\%)$ & 100 & 7.69 & 53.08 & $\mathrm{~N}$ \\
\hline
\end{tabular}

Abbreviations: Cyclophosph., Cyclophosphamide; I. steroids, Intra-tympanic steroids; N, No; S. steroids, Systemic steroids; Y, Yes. 
Table 3 Initial audiometric characteristics from 16 affected ears at the time of admission to our service

\begin{tabular}{|c|c|c|c|c|c|}
\hline Case & Affected Side & Degree of Initial Loss & Initial Audio Curve & Initial SRI (dB) & $\begin{array}{l}\text { Initial SRI } \\
\text { Monosyllable (\%) }\end{array}$ \\
\hline 1 & $\mathrm{R}$ & moderate & downward & up to 25 & 92 \\
\hline $2^{*}$ & $\mathrm{~L}$ & deep & NA & $>90$ & 0 \\
\hline $2^{*}$ & $\mathrm{R}$ & deep & downward & $>90$ & 0 \\
\hline 3 & $\mathrm{~L}$ & severe & flat & $>90$ & 0 \\
\hline 4 & $\mathrm{~L}$ & deep & U & $>90$ & 0 \\
\hline $5^{*}$ & $\mathrm{R}$ & severe & downward & $>90$ & 76 \\
\hline $5^{*}$ & $\mathrm{~L}$ & moderate & flat & 26 to 40 & 44 \\
\hline 6 & $\mathrm{R}$ & deep & flat & $>90$ & 0 \\
\hline 7 & $\mathrm{R}$ & severe & U & $>90$ & 0 \\
\hline 8 & $\mathrm{R}$ & light & downward & 26 to 40 & 96 \\
\hline 9 & $\mathrm{R}$ & deep & NA & $>90$ & 0 \\
\hline
\end{tabular}

Abbreviations: L, Left; R, Right; SRI, speech recognition index.

* Bilateral cases.

we could not classify the type of curve because the loss was too profound (-Table 3 ).

At the end of follow-up, the degree of hearing loss of the affected ears presented as follows: seven (43.75\%) ears with profound loss, two (12.25\%) with severe loss, four (25.00\%) with moderate loss, two (12.25\%) with mild hearing loss, and one $(6.25 \%)$ showed no hearing loss (-Table 4 ).

The RTS at the end of follow-up of affected ears presented as follows: RTS greater than $90 \mathrm{~dB}$ in seven (43.75\%), RTS between 71-90 dB in one (6.25\%), RTS between 41-70 dB five
(31.25\%), RTS between 26 and $40 \mathrm{~dB}$ in one (6.25\%), and RTS below $25 \mathrm{~dB}$ in two (12.25\%) (-Table 4). In four $(25.00 \%$ ) ears of different patients, there was an improvement greater than $40 \%$ of the SRI after treatment. In both ears (12.50\%) of patients with Cogan's syndrome, the worsening index was higher than $40 \%$.

Regarding responsiveness to corticosteroids, seven ears (43.75\%) showed at least a partial or temporary response to this treatment. This response was observed by improving audiometric thresholds. In these seven ears, the time between

Table 4 Audiometric characteristics from 16 affected ears at the end of follow-up

\begin{tabular}{|l|l|l|l|l|}
\hline Case & Affected Side & Degree Final Loss & Final RST (dB) & $\begin{array}{l}\text { SRI Final } \\
\text { Monosyllable (\%) }\end{array}$ \\
\hline 1 & R & severe & 41 to 70 & 75 \\
\hline $2^{*}$ & L & deep & $>90$ & 0 \\
\hline $2^{*}$ & R & deep & $>90$ & 12 \\
\hline 3 & L & moderate & 41 to 70 & 56 \\
\hline 4 & L & deep & $>90$ & 0 \\
\hline $5^{*}$ & R & deep & $>90$ & 0 \\
\hline $5^{*}$ & L & deep & $>90$ & 0 \\
\hline 6 & R & severe & 71 to 90 & 60 \\
\hline 7 & R & moderate & 41 to 70 & 76 \\
\hline 8 & R & light & 26 to 40 & 96 \\
\hline 9 & R & moderate & 41 to 70 & 0 \\
\hline 10 & R & no loss & up to 25 & 100 \\
\hline $11^{*}$ & R & deep & 0 \\
\hline $11^{*}$ & L & moderate & 41 to 70 & 95 \\
\hline 12 & L & deep & $>90$ & 0 \\
\hline 13 & R & light & up to 25 & 100 \\
\hline
\end{tabular}

Abbreviations: L, Left; R, Right; RTS, reception threshold speech; SRI, speech recognition index.

* bilateral cases. 
Table 5 Responsiveness to treatment, support of the response and comparison between the initial and final audiometric characteristics of 16 affected ears

\begin{tabular}{|l|l|l|l|l|l|l|l|l|}
\hline Case & $\begin{array}{l}\text { Affected } \\
\text { Side }\end{array}$ & $\begin{array}{l}\text { Response } \\
\text { to } \\
\text { steroids }\end{array}$ & $\begin{array}{l}\text { Time after } \\
\text { starting } \\
\text { medication } \\
\text { (days) }\end{array}$ & $\begin{array}{l}\text { Sustained response } \\
\text { or later worsens } \\
\text { (months) }\end{array}$ & $\begin{array}{l}\text { Degree } \\
\text { of initial } \\
\text { loss }\end{array}$ & $\begin{array}{l}\text { Degree } \\
\text { of final } \\
\text { loss }\end{array}$ & $\begin{array}{l}\text { SRI-initial } \\
\text { monosyllable } \\
\text { (\%) }\end{array}$ & $\begin{array}{l}\text { SRI-final } \\
\text { monosyllable } \\
\text { (\%) }\end{array}$ \\
\hline 1 & $\mathrm{R}$ & $\mathrm{Y}$ & 24 & 9 (subsequent worsening) & moderate & severe & 92 & 75 \\
\hline $2^{*}$ & $\mathrm{~L}$ & $\mathrm{~N}$ & not occurred & & deep & deep & 0 & 0 \\
\hline $2^{*}$ & $\mathrm{R}$ & $\mathrm{N}$ & not occurred & & deep & deep & 0 & 12 \\
\hline 3 & $\mathrm{~L}$ & $\mathrm{Y}$ & 15 & 20 (tracking ended) & severe & moderate & 0 & 0 \\
\hline 4 & $\mathrm{~L}$ & $\mathrm{~N}$ & not occurred & & deep & deep & 0 & 56 \\
\hline $5^{*}$ & $\mathrm{R}$ & $\mathrm{N}$ & not occurred & & severe & deep & 75 & 0 \\
\hline $5^{*}$ & $\mathrm{~L}$ & $\mathrm{~N}$ & not occurred & & moderate & deep & 45 & 0 \\
\hline 6 & $\mathrm{R}$ & $\mathrm{Y}$ & 60 & 7 (worsening afterwards) & deep & severe & 0 & 0 \\
\hline 7 & $\mathrm{R}$ & $\mathrm{Y}$ & 5 & 2 (tracking ended) & severe & moderate & 0 & 60 \\
\hline 8 & $\mathrm{R}$ & $\mathrm{N}$ & not occurred & & light & light & 96 & 76 \\
\hline 9 & $\mathrm{R}$ & $\mathrm{Y}$ & 20 & 9 (tracking ended) & deep & moderate & 0 & 96 \\
\hline 10 & $\mathrm{R}$ & $\mathrm{Y}$ & 15 & 2 (tracking ended) & severe & no loss & 0 & 0 \\
\hline $11^{*}$ & $\mathrm{R}$ & $\mathrm{N}$ & not occurred & & deep & deep & 0 & 100 \\
\hline $11^{*}$ & $\mathrm{~L}$ & $\mathrm{Y}$ & 12 & 16 (tracking ended) & moderate & moderate & 92 & 0 \\
\hline 12 & $\mathrm{~L}$ & $\mathrm{~N}$ & not occurred & & deep & deep & 0 & 95 \\
\hline 13 & $\mathrm{R}$ & $\mathrm{N}$ & not occurred & & light & light & 92 & 0 \\
\hline
\end{tabular}

Abbreviations: L, Left; N, No; R, Right; SRI, speech recognition index; Y, Yes.

* bilateral cases.

the beginning of medication and hearing improvement ranged from 5 to 60 days, with an average of 21.6 days. In five of these ears ( $31 \%$ of the sample), the answer remained sustained until the last monitoring conducted with these patients. In two ears of different cases initially responsive to corticosteroid treatment, the improvement held for about eight months; after this period, however, there was a further

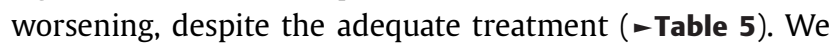
found normal hearing thresholds in only one ear of a case (7.69\%) with unilateral involvement.

Out of the other nine ears (56.25\%), which did not respond to the treatment ( $\mathbf{- T a b l e ~} \mathbf{5}$ ), seven $(77.77 \%$ ) remained unchanged with audiometric thresholds during evolution. Five (71.42\%) had profound loss from beginning to end monitoring. Both ears of the patient with Cogan's syndrome showed rapidly irreversible progressive audiometric worsening.

At the end of follow-up, according to the hearing improvement criteria adopted, of the 16 affected ears, five (31.25\%) showed improvement, three (18.75\%) got worse, and eight (50.00\%) had the final loss similar to the initial loss. Note that, initially, two more ears (12.5\%), in addition to the five aforementioned, showed a temporary hearing improvement, with deterioration of the parameters evaluated during evolution (-Table 5).

\section{Discussion}

Sudden sensorineural hearing loss is a medical emergency and understanding its possible etiologies for indication of a more effective treatment is a goal that remains under pursuit by the medical and scientific communities. The probable cause immune-mediated is suggested by many specialists; so, due to its possible responsiveness when set up an appropriate and early treatment, should always be remembered.

It is important to highlight that sudden SNHL is a clinical entity defined by hearing loss symptom of sudden onset associated with audiological and temporal criteria, while the immune-mediated SNHL would be a likely etiologic diagnosis.

Of our sample of 339 patients with sudden SNHL, 13 (3.83\%) had confirmed SAD. Whereas this systemic immune-mediated change is a possible cause of hearing loss in such patients, according to the literature, only 10 to $29 \%$ of sudden SNHL have a defined etiology. ${ }^{12,24}$ Given the difficulty to confirm the diagnosis, and mainly the lack of criteria for this diagnosis, we chose not to include possible immunemediated hearing loss, which are not associated with SAD but confirmed with rheumatologic criteria and laboratory tests in this analysis. This way, the prevalence of sudden sensorineural hearing loss with immune-mediated etiology should be even greater.

In this study, the mean age of patients with SAD at the time of SSNHL installation was 44 years and the same average was found in the sample without SAD. This is in agreement with the literature, in which several studies show that both sudden SNHL and immune-mediated SNHL most often affect patients between their thirties and fifties. ${ }^{17,18}$

Regarding gender, most patients in our sample were women (69.23\%). This was expected, because, according to Cooper and Stroehla, ${ }^{25} 80 \%$ of patients with SAD are women. 
In 1988 , Hughes et $\mathrm{al}^{19}$ showed that $65 \%$ of patients with immune-mediated hearing loss were female. Sudden SNHL not associated with the SAD, though, affected both sexes in the same proportion, which is also supported by the literature. $^{26}$ In other words, with regard to the prevalence of gender, our sample meets a typical feature of immunemediated SNHL, not of idiopathic sudden sensorineural hearing loss.

Regarding ethnicity, in our sample, $61.54 \%$ were Caucasian and $38.46 \%$ were afro-descendant. The predominance of white skin color is noteworthy; however, we did not find this correlation in the literature.

The immune-mediated SNHL can be associated with SAD in $30 \%$ of cases. However, the incidence of involvement of the inner ear in the SAD is very variable. ${ }^{19,20}$ Installing this hearing loss can present abruptly, featuring a sudden SNHL, and the progression can be fast and with bilateral involvement.

The pathophysiology of immune-mediated character that may be involved in inner ear dysfunction include: immediate hypersensitivity, with production of IgE immunoglobulins against cochlear antigens; immune complex deposits in the stria vascularis and spiral ligament; direct action of cytotoxic $\mathrm{T}$ cells in the cochlea; and delayed hypersensitivity with immune reactivity mediated collagen type II. These mechanisms can act in a complementary way and often do so simultaneously. ${ }^{27}$

Although some authors report that hearing loss can manifest as the first symptom of SAD, ${ }^{28}$ this was not the case in our sample. All patients had already had other systemic symptoms related to autoimmune disease prior to the installation of sudden SNHL.

In our sample, $30.77 \%$ had SLE, the same prevalence as that of patients with RA.

Several authors have demonstrated the relationship between SLE and sudden SNHL. Researchers have also speculated on the existence of a strong relationship between the presence of anticardiolipin antibodies, often found in patients with SLE, and sudden SNHL. 27,29 In such cases, the authors argue that the formation of microthrombus in cochlear vessels could be the etiology of sudden SNHL.

In a retrospective cohort population study in patients with SLE in the population of Taiwan, Lin et $\mathrm{al}^{5}$ reinforce the relationship between SLE and sudden SNHL by showing that patients affected by this immune-mediated disease have an incidence rate of sudden SNHL up to 4.27 times higher than the general population.

The literature related to RA with hearing $\operatorname{loss}^{30}$ demonstrates involvement of the inner ear in $60 \%$ of cases. Moreover, although we believe in this association, we found no studies that indicate a relationship of this disease with sudden SNHL.

In our study, $15.38 \%$ of patients had scleroderma. Hearing loss and other audiological symptoms such as tinnitus, have been reported in up to $40 \%$ of patients with systemic sclerosis. ${ }^{31}$ Deroee et al $^{4}$ described the case of a 65 year-old patient who developed sudden SNHL as the first manifestation of scleroderma. The two patients with scleroderma shown in our study showed sudden SNHL years after diagnosis of the disease.

One of the patients included in this study had Sjogren's syndrome. The literature shows the presence of hearing loss in up to $25 \%$ of patients with the syndrome. ${ }^{32}$ Nonetheless, we have not found in our review any article that would correlate this syndrome with sudden SNHL. Thus, our report renders it a breakthrough.

Yet another patient included in this study has presented with Cogan's syndrome. Several authors associate this syndrome with sudden SNHL. ${ }^{33-35}$

Another patient included in our sample had Susac syndrome. The literature review shows that many authors correlate this devastating syndrome with sudden SNHL. ${ }^{36-39}$

One of the patients included in our case series was diagnosed with psoriasis. We found little evidence in the literature showing a possible link between this disease and sudden SNHL. ${ }^{40}$

The vitiligo patient described in our results also had RA. Several authors have described increased prevalence of SNHL, as well as other hearing disorders in patients with vitiligo. ${ }^{41}$ However, we have not found any article that directly relates vitiligo with sudden sensorineural hearing loss.

One of our patients had ankylosing spondylitis (AS). In our review, we found evidence of an increased prevalence of sensorineural hearing loss in patients with AS, but not specifically its relationship with sudden SNHL. ${ }^{42}$

Eight patients (61.53\%) expressed other systemic comorbidities aside from SAD, at the time when they had sudden SNHL: hypertension, diabetes mellitus, CRF, HT, UA, and smoking. It is not sure that these comorbidities may be risk factors for sudden SNHL. ${ }^{43}$

Regarding associated symptoms, all (100\%) patients in our study had tinnitus; 54\%, dizziness, and 38\%, ear fullness concomitant with sudden sensorineural hearing loss. These percentages are identical to those found in the literature concerning the sudden SNHL idiopathic. ${ }^{44}$ As for immunemediated hearing loss, $83 \%$ expressed tinnitus; $60 \%$ dizziness, and $50 \%$ ear fullness. ${ }^{45,46}$ In other words, we found a higher incidence of tinnitus in our study when compared with literature findings.

The otoscopy of 13 patients did not show significant alterations. This matches findings in the literature, noting the normality of these patients' tests. ${ }^{17}$

In our study, we chose not to emphasize the analysis of autoantibodies, as the diagnosis of several autoimmune diseases evaluated would require several specific laboratory tests whose results would vary at different stages of evolution for each patient. The diagnosis for each of the above diseases relies on their own rheumatologic clinical criteria. Regarding the search for specific autoantibodies of the inner ear for the diagnosis of immune-mediated SNHL, although once considered a promising method, recent studies show a low sensitivity of these tests. Among these, the most researched is the Anti-Heat Shock Protein 70 antibody (Hsp 70), which also has not demonstrated efficiency in diagnosing such illness. ${ }^{23}$

Out of the 13 patients, ten (76.92\%) underwent magnetic resonance imaging. Cochlear showed no enhancement in any 
of the ears on the T1-weighted after contrast injection. Fitzgerald and Mark described a series of cases in which $66 \%$ of patients with sudden SNHL with immune-mediated etiology presented highlight the labyrinth after contrast injection. Although post-administration gadolinium enhancement of the inner ear is a widespread finding in the literature, we found scarce studies confirming the hypothesis of immune-mediated SNHL. These were based on a few cases that demonstrate this alteration. ${ }^{22,47}$ We believe the discrepancy between our findings and the literature could be justified with examination after the start of corticosteroids therapy and the methodology of accomplishment and different analysis.

The currently recommended treatment for sudden SNHL is the use of systemic corticosteroids in full dose, which is the equivalent of $1 \mathrm{mg} / \mathrm{kg}$ of prednisone, and a gradual reduction of the dose based on audiometric recovery parameters. Transtympanic infusion of corticosteroids is indicated in cases of failure or contraindication of systemic therapy or incomplete improves as a rescue therapy. ${ }^{48}$ In cases of immune-mediated SNHL, in addition to systemic corticosteroids and/or transtympanic, it is possible to use other immunosuppressive medications. These are recommended for patients who did not respond to corticosteroids or as a way to preserve the prolonged use of corticosteroids to provide sustainability and maintain hearing recovery, as well as prevent and slow the deterioration of hearing of these individuals. ${ }^{17,46,49}$

All cases presented in this study had full dose systemic corticosteroids as initial treatment for sudden SNHL. Only a particular person (case 1) was treated with a series of transtympanic injections of corticosteroids and, therefore, proved to be refractory to the initial systemic therapy.

As all of the patients had SAD, the use of other immunesuppressive medications was very common, occurring in $77 \%$ of cases during follow-up at our clinic. Sometimes we indicated such medications for the treatment of hearing loss; in most cases, however, it was aimed to control the systemic disease base.

The most commonly used immunosuppressant in the cases was methotrexate, administered to $40 \%$ of patients. Its efficacy in the treatment of immune-mediated sensorineural hearing loss has been widely studied. More recently, however, a study demonstrated that this drug does not have the expected result for the treatment of immune-mediated sensorineural hearing loss. ${ }^{49}$ Garcia-Berrocal et al ${ }^{50}$ reported the treatment of five patients with methotrexate for refractory immune-mediated SNHL. According to the authors, the drug improved vestibular symptoms but was not successful in achieving hearing improvement. Although we initially believed in the effectiveness of methotrexate, we have not seen significant improvement to our patients hearing.

Among other immunosuppressants used in patients followed, the literature still points to cyclophosphamide as an alternative for patients refractory to corticosteroids or the ones who have contraindication to use it. However, we must be alert to the possible ototoxic effects of this drug, which can lead to worsening of hearing. ${ }^{46}$ Two patients described in our study used this medication and we did not detect ototoxic effect during either one's follow-up.

We have not found in our review overwhelming evidence that shows that chloroquine, azathioprine, or sulfasalazine, also used in the patients described above, are effective in the treatment of SNHL immune mediated. ${ }^{49}$

One of our patients tried infliximab for a period. We did not notice improvement in hearing parameters during his use of the medication. Liu et $\mathrm{al}^{51}$ demonstrated eight cases of patients with immune-mediated SNHL refractory to corticosteroid treated with infliximab. None of them showed audiometric improvement.

Although controversy remains over the effectiveness of these immunosuppressive drugs for the hearing improvement, we believe the specific treatment to control SAD can indeed slow down the progression of the inner ear lesion. Thus, even if there is recovery of hearing thresholds already committed, it would prevent the progression of the lesion in the ear as well as contralateral involvement. Moreover, we observed significant improvement of vestibular symptoms during the use of these drugs. The literature also describes the improvement of vestibular symptoms with the use of immunosuppressants, even without achieving hearing improvement. ${ }^{50}$

Patients with sudden SNHL with probable immune-mediated origin, as opposed to those with idiopathic sudden SNHL without the suspicion, have more severe initial impairment, higher percentage of bilateral, lower response to treatment, and worse prognosis. This is likely to occur due to the continued assault on the immune-mediated inner ear structures, whereas most patients with idiopathic sudden SNHL suffer a single, acute triggering event, without further worsening of hearing loss. Several authors have also stated that the responsiveness of immune-mediated SNHL happens in the early stages, that is, in the first days or weeks. ${ }^{16,19}$ Therefore, establishing appropriate treatment at the earliest possible time is important to be able to stop the progression of the lesion and get a better prognosis. ${ }^{49}$

In our study, the time elapsed between the onset of sudden SNHL and the start of treatment was 21 days on average. We believe that this delay occurred due to other priorities demanded by SAD, which means some patients do not prioritize the treatment of sudden sensorineural hearing loss among the systemic manifestations caused by the underlying disease. Several authors show the correlation between the start of treatment time and the prognosis for hearing recovery in cases of sudden SNHL and suggest that the shorter it takes, the better the prognosis. ${ }^{52}$

The mean follow-up time of these patients was 27.6 months, which provided us a longitudinal observation of their evolution. We realize, therefore, the dynamic and often unpredictable nature of the illness, even with treatment for the underlying disease. One should also pay attention to further worsening of hearing loss; it may be a sign of acute relapse or activity of the disease base. ${ }^{30}$

As for unilateral or bilateral involvement, out of the 13 cases presented, three (23\%) had bilateral involvement, including one occurring simultaneously. Curiously enough, in these three cases, the installation of hearing loss happened 
suddenly in both ears. We believe that bilateral involvement occurred in only $23 \%$ of cases in our sample because we established correct treatment early and patients underwent rigorous clinical monitoring. As the bilateral involvement is often asymmetric, the other ten patients may subsequently have a contralateral involvement that can be installed both sudden way of progressively. We did not find in the literature the incidence of bilateral involvement in cases of immunemediated SNHL in patients with SAD.

Bilateral involvement is a common feature of the SNHL immune-mediated, even used as an indicator for diagnostic. ${ }^{16}$ However, in our view, bilateral is clearly predominant in undiagnosed patients who have not received proper treatment. When we think of sudden SNHL as a whole, the literature describes $1.7 \%$ to $3 \%$ of bilateral occurrences, being the exception rather than the rule. ${ }^{26}$ Fetterman et al ${ }^{26}$ describe patients with simultaneous bilateral sudden SNHL as well as symmetrical audiometric curves between both sides. The authors state they are more likely to have positive antinuclear antibodies, indicating an association with immune-mediated etiology.

The patient with bilateral simultaneous sudden SNHL received diagnosis of SLE as the underlying disease. It was quite distressing for both the patient and his physician to face bilateral and simultaneous installation. ${ }^{17}$ Nevertheless, this was the patient inspired our search for a specific treatment for hearing loss (60 days). He was in a severe state of depression compounded by hearing loss.

In $70 \%$ of ears, the degree of hearing loss present upon admission to our study was severe or profound: $43.75 \%$ had profound hearing loss while $25 \%$ had severe hearing loss. Penido et $\mathrm{al}^{44}$ reported that $50 \%$ of patients with sudden SNHL analyzed in their clinic had severe or profound loss. Initially, $63 \%$ of ears in our sample presented RTS greater than $90 \mathrm{~dB}$ and SRI zero. We ponder that the higher incidence of more severe early hearing loss in our current sample results from a more aggressive lesion due to the likely immune-mediated etiology involved. These initial audiometric findings were indication of a worse prognosis from the beginning.

Our patients did not show a predominant audiometric curve configuration at the time of admission. We stress that in $25 \%$ of affected ears, we could not classify the type of audiometric curve because the loss was too deep. The literature shows that $43 \%$ of patients with sudden sensorineural hearing loss showed plane curve and the type of audiometric initial curve is not related to the prognosis of these patients. ${ }^{44}$ Some authors present results suggesting the involvement fist of higher frequencies in immune-mediated SNHL, however, this fact was not observed in our patients.

As for responsiveness to corticosteroids, 44\% of affected ears presented a presented at least a partial or temporary positive response to this treatment, with improvement of the audiometric thresholds. This data agrees with the literature, whereby several studies report responsiveness to corticosteroids at a rate ranging from $44 \%$ to $70 \%$ in cases of immunemediated SNHL. ${ }^{45,53}$

In our study, $31 \%$ of the ears analyzed showed sustained response to the latest monitoring conducted. However, two ears (12.5\%) of different patients sustained a response for about eight months, after which they had hearing impairment despite treatment. According to Broughton et al, ${ }^{45}$ $70 \%$ of patients with immune-mediated SNHL are initially responsive to corticosteroids and only $14 \%$ sustain this response after 34 months of treatment.

Although we have not monitored for as long as this author, we believe that the response to corticosteroids may be temporary in some cases, a fact that brings us great concern.

Still about the responsiveness to treatment, we found normal hearing thresholds only in one ear of one case (7.69\%) with unilateral involvement, suggesting a poor prognosis of this disease. Another fact that also strongly indicates a poor prognosis related to immune-mediated SNHL associated in patients with SAD is that, at the end of follow-up, only $30 \%$ showed sustained improvement, while $20 \%$ got worse, and $50 \%$ had a final loss similar to the initial loss. Unfortunately, we know that those who improved could still worsen and it is unlikely those who suffered worsening will improve satisfactorily. Moreover, some ears remained stable: 71\% showed profound loss from beginning to end of monitoring. We believe that this occurs because there is a point at which damage to the inner ear cells becomes irreversible and, therefore, there is no possibility of improvement with treatment currently available.

The likely immune-mediated etiology of sensorineural hearing loss is still uncertain, as well as the association with systemic autoimmune diseases, more research is needed to clarify these possible relationships. In the future, with the new possibilities of molecular medicine, gene therapy, and the development of treatment with stem cells, this reality can change and give us the ability to offer a better prognosis for such patients.

\section{Conclusion}

In our sample of patients with sudden sensorineural hearing loss, the prevalence of systemic autoimmune disease was considerable. This association was more frequent among white adult women. Most patients had unilateral hearing loss, severe or profound, associated with concomitant dizziness. All had tinnitus. Most cases did not improve audiometric thresholds, not even with the treatment.

Our patients with sudden SNHL and SAD have more severe initial impairment, higher percentage of bilateral, lower response to treatment, and worse prognosis when compared with patients without this association.

\section{References}

1 Toubi E, Ben-David J, Kessel A, Halas K, Sabo E, Luntz M. Immunemediated disorders associated with idiopathic sudden sensorineural hearing loss. Ann Otol Rhinol Laryngol 2004;113(6): 445-449

2 Compadretti GC, Brandolini C, Tasca I. Sudden sensorineural hearing loss in lupus erythematosus associated with antiphospholipid syndrome: case report and review. Ann Otol Rhinol Laryngol 2005;114(3):214-218 
3 Kang KT, Young YH. Sudden sensorineural hearing loss in a patient with primary antiphospholipid syndrome. J Laryngol Otol 2008; 122(2):204-206

4 Deroee AF, Huang TC, Morita N, Hojjati M. Sudden hearing loss as the presenting symptom of systemic sclerosis. Otol Neurotol 2009; 30(3):277-279

5 Lin C, Lin SW, Weng SF, Lin YS. Risk of sudden sensorineural hearing loss in patients with systemic lupus erythematosus: a population-based cohort study. Audiol Neurootol 2013;18(2): 95-100

6 Wilson WR, Byl FM, Laird N. The efficacy of steroids in the treatment of idiopathic sudden hearing loss. A double-blind clinical study. Arch Otolaryngol 1980;106(12):772-776

7 Byl FM Jr. Sudden hearing loss: eight years' experience and suggested prognostic table. Laryngoscope 1984;94(5 Pt 1):647-661

8 Simmons FB. Theory of membrane breaks in sudden hearing loss. Arch Otolaryngol 1968;88(1):41-48

9 Schuknecht HF, Kimura RS, Naufal PM. The pathology of sudden deafness. Acta Otolaryngol 1973;76(2):75-97

10 Wilson WR, Veltri RW, Laird N, Sprinkle PM. Viral and epidemiologic studies of idiopathic sudden hearing loss. Otolaryngol Head Neck Surg 1983;91(6):653-658

11 Berrocal JR, Ramírez-Camacho R. Sudden sensorineural hearing loss: supporting the immunologic theory. Ann Otol Rhinol Laryngol 2002;111(11):989-997

12 Chau JK, Lin JR, Atashband S, Irvine RA, Westerberg BD. Systematic review of the evidence for the etiology of adult sudden sensorineural hearing loss. Laryngoscope 2010;120(5):1011-1021

13 Penido NO, Aumond MD, Leonhardt FD, Abreu CEC, Toledo RN. Disacusia neurossensorial imunomediada. Rev Bras Otorrinolaringol (Engl Ed) 2002;68:730-734

14 Lehnhardt E. [Sudden hearing disorders occurring simultaneously or successively on both sides]. Z Laryngol Rhinol Otol 1958;37(1): $1-16$

15 Schiff M, Brown M. Hormones and sudden deafness. Laryngoscope 1974;84(11):1959-1981

16 McCabe BF. Autoimmune sensorineural hearing loss. Ann Otol Rhinol Laryngol 1979;88(5 Pt 1):585-589

17 Alvarenga EHL, Costa SS, Cruz OLM. Disacusia Neurossensorial Imunomediada. In: Cruz OLM, Costa SS eds. Otologia Clínica e Cirúrgica. Rio de Janeiro, Brazil: Revinter; 2000:307-13

18 Bovo R, Ciorba A, Martini A. The diagnosis of autoimmune inner ear disease: evidence and critical pitfalls. Eur Arch Otorhinolaryngol 2009;266(1):37-40

19 Hughes GB, Barna BP, Kinney SE, Calabrese LH, Nalepa NJ. Clinical diagnosis of immune inner-ear disease. Laryngoscope 1988;98(3): 251-253

20 Agrup C, Luxon LM. Immune-mediated inner-ear disorders in neuro-otology. Curr Opin Neurol 2006;19(1):26-32

21 Mazlumzadeh M, Lowe VJ, Mullan BP, Fabry DA, McDonald TJ, Matteson EL. The utility of positron emission tomography in the evaluation of autoimmune hearing loss. Otol Neurotol 2003;24(2): 201-204

22 Teszler CB, Williams MT, Belange G, Ayache D. Labyrinthitis related to Wegener granulomatosis: magnetic resonance imaging findings. Otol Neurotol 2008;29(5):721-722

23 Ianuale C, Cadoni G, De Feo E, et al. A systematic review and metaanalysis of the diagnostic accuracy of anti-heat shock protein 70 antibodies for the detection of autoimmune hearing loss. Otol Neurotol 2013;34(2):214-219

24 Penido NO, Cruz OL, Zanoni A, Inoue DP. Classification and hearing evolution of patients with sudden sensorineural hearing loss. Braz J Med Biol Res 2009;42(8):712-716

25 Cooper GS, Stroehla BC. The epidemiology of autoimmune diseases. Autoimmun Rev 2003;2(3):119-125

26 Fetterman BL, Luxford WM, Saunders JE. Sudden bilateral sensorineural hearing loss. Laryngoscope 1996;106(11):1347-1350
27 Cecatto SB, Garcia RID, Costa KS, Anti SMA, Longone E, Rapoport PB. Perda auditiva sensorioneural no lúpus eritematoso sistêmico: relato de três casos. Rev Bras Otorrinolaringol (Engl Ed) 2004; 70:398-403

28 Green L, Miller EB. Sudden sensorineural hearing loss as a first manifestation of systemic lupus erythematosus: association with anticardiolipin antibodies. Clin Rheumatol 2001;20(3): 220-222

29 Hisashi K, Komune S, Taira T, Uemura T, Sadoshima S, Tsuda H. Anticardiolipin antibody-induced sudden profound sensorineural hearing loss. Am J Otolaryngol 1993;14(4):275-277

30 Takatsu M, Higaki M, Kinoshita H, Mizushima Y, Koizuka I. Ear involvement in patients with rheumatoid arthritis. Otol Neurotol 2005;26(4):755-761

31 Kastanioudakis I, Ziavra N, Politi EN, Exarchakos G, Drosos AA, Skevas A. Hearing loss in progressive systemic sclerosis patients: a comparative study. Otolaryngol Head Neck Surg 2001;124(5): 522-525

32 Montoya-Aranda IM, Peñaloza-López YR, Gutiérrez-Tinajero DJ. [Sjögren's syndrome: Audiological and clinical behaviour in terms of age]. Acta Otorrinolaringol Esp 2010;61(5): 332-337

33 Gluth MB, Baratz KH, Matteson EL, Driscoll CL. Cogan syndrome: a retrospective review of 60 patients throughout a half century. Mayo Clin Proc 2006;81(4):483-488

34 Orsoni JG, Zavota L, Pellistri I, Piazza F, Cimino L. Cogan syndrome. Cornea 2002;21(4):356-359

35 Cote DN, Molony TB, Waxman J, Parsa D. Cogan's syndrome manifesting as sudden bilateral deafness: diagnosis and management. South Med J 1993;86(9):1056-1060

36 Kleffner I, Duning T, Lohmann H, et al. A brief review of Susac syndrome. J Neurol Sci 2012;322(1-2):35-40

37 Gross M, Eliashar R. Update on Susac's syndrome. Curr Opin Neurol 2005;18(3):311-314

38 Bitra RK, Eggenberger E. Review of Susac syndrome. Curr Opin Ophthalmol 2011;22(6):472-476

39 Ozturk A, Degirmenci Y, Tunc M, Kececi H. Susac's syndrome: a case of simultaneous development of all three components of the triad. J Neurol Sci 2013;324(1-2):187-189

40 Srikumar S, Deepak MK, Basu S, Kumar BN. Sensorineural hearing loss associated with psoriatic arthritis. J Laryngol Otol 2004; 118(11):909-911

41 Akay BN, Bozkir M, Anadolu Y, Gullu S. Epidemiology of vitiligo, associated autoimmune diseases and audiological abnormalities: Ankara study of 80 patients in Turkey. J Eur Acad Dermatol Venereol 2010;24(10):1144-1150

42 Kahveci OK, Demirdal US, Duran A, Altuntas A, Kavuncu V, Okur E. Hearing and cochlear function of patients with ankylosing spondylitis. Clin Rheumatol 2012;31(7):1103-1108

43 Aimoni C, Bianchini C, Borin M, et al. Diabetes, cardiovascular risk factors and idiopathic sudden sensorineural hearing loss: a case-control study. Audiol Neurootol 2010;15(2): 111-115

44 Penido NdeO, Ramos HVL, Barros FA, Cruz OLM, Toledo RN. Clinical and etiological factors and evolution of hearing in sudden deafness. Braz J Otorhinolaryngol 2005;71(5):633-638

45 Broughton SS, Meyerhoff WE, Cohen SB. Immune-mediated inner ear disease: 10-year experience. Semin Arthritis Rheum 2004; 34(2):544-548

46 Ruckenstein MJ. Autoimmune inner ear disease. Curr Opin Otolaryngol Head Neck Surg 2004;12(5):426-430

47 Zavod MB, Sataloff RT, Rao VM. Frequency of cochlear enhancement on magnetic resonance imaging in patients with autoimmune sensorineural hearing loss. Arch Otolaryngol Head Neck Surg 2000;126(8):969-971

48 Stachler RJ, Chandrasekhar SS, Archer SM, et al; American Academy of Otolaryngology-Head and Neck Surgery. Clinical practice 
guideline: sudden hearing loss. Otolaryngol Head Neck Surg 2012; 146(3, Suppl)S1-S35

49 Buniel MC, Geelan-Hansen K, Weber PC, Tuohy VK. Immunosuppressive therapy for autoimmune inner ear disease. Immunotherapy 2009;1(3):425-434

50 García-Berrocal JR, Ibáñez A, Rodríguez A, et al. Alternatives to systemic steroid therapy for refractory immune-mediated inner ear disease: A physiopathologic approach. Eur Arch Otorhinolaryngol 2006;263(11):977-982
51 Liu YC, Rubin R, Sataloff RT. Treatment-refractory autoimmune sensorineural hearing loss: response to infliximab. Ear Nose Throat J 2011;90(1):23-28

52 Chang NC, Ho KY, Kuo WR. Audiometric patterns and prognosis in sudden sensorineural hearing loss in southern Taiwan. Otolaryngol Head Neck Surg 2005;133(6):916-922

53 Zeitoun H, Beckman JG, Arts HA, et al. Corticosteroid response and supporting cell antibody in autoimmune hearing loss. Arch Otolaryngol Head Neck Surg 2005;131(8):665-672 